PROCEEDINGS OF THE

AMERICAN MATHEMATICAL SOCIETY

Volume 129, Number 5, Pages 1545-1549

S 0002-9939(00)05676-8

Article electronically published on October 20, 2000

\title{
MINIMAL NUMBER OF SINGULAR FIBERS IN A LEFSCHETZ FIBRATION
}

\author{
MUSTAFA KORKMAZ AND BURAK OZBAGCI \\ (Communicated by Ronald A. Fintushel)
}

\begin{abstract}
There exists a (relatively minimal) genus $g$ Lefschetz fibration with only one singular fiber over a closed (Riemann) surface of genus $h$ iff $g \geq 3$ and $h \geq 2$. The singular fiber can be chosen to be reducible or irreducible. Other results are that every Dehn twist on a closed surface of genus at least three is a product of two commutators and no Dehn twist on any closed surface is equal to a single commutator.
\end{abstract}

\section{INTRODUCTION}

A Lefschetz fibration is a fibration of a smooth 4-manifold over a surface with general fiber another closed orientable surface, which may admit certain singular fibers. The isomorphism class of a Lefschetz fibration is determined by its global monodromy. This leads to a combinatorial approach to study the topology of smooth 4-manifolds (which admit Lefschetz fibrations) by their monodromy representations in the mapping class groups. In particular, the number of singular fibers in a Lefschetz fibration cannot be arbitrary and depends on the genus of the fiber, the genus of the base and the algebraic structure of the mapping class group of the generic fiber - as we will illustrate below.

Let $N(g, h)$ denote the minimal number of singular fibers in a relatively minimal genus $g$ Lefschetz fibration (with at least one singular fiber) over a closed (Riemann) surface of genus $h$.

Our first result is the following theorem.

Theorem 1.1. $N(g, h)=1$ iff $g \geq 3$ and $h \geq 2$.

Let $\Sigma_{g}$ be a closed connected orientable surface of genus $g$. The mapping class group $\mathcal{M}_{g}$ of $\Sigma_{g}$ is defined to be the group of isotopy classes of orientation preserving diffeomorphisms $\Sigma_{g} \rightarrow \Sigma_{g}$.

For a simple closed curve $a$ on an oriented surface, let us denote by $t_{a}$ the right Dehn twist about $a$.

We also prove the following theorem, which is needed for the proof of Theorem 1.1.

Received by the editors February 26, 1999 and, in revised form, July 28, 1999.

1991 Mathematics Subject Classification. Primary 57M99; Secondary 20F38.

Key words and phrases. Lefschetz fibrations, 4-manifolds, mapping class groups.

(C)2000 American Mathematical Society 
Theorem 1.2. Let $\Sigma_{g}$ be a closed connected oriented surface of genus $g$ and let a be a simple closed curve on $\Sigma_{g}$. If $g \geq 3$, then $t_{a}$ can be written as a product of two commutators.

\section{Definitions AND PROOFs}

Definition 2.1. Let $M$ be a closed, connected, oriented smooth four manifold. A Lefschetz fibration is a smooth map $\pi: M \rightarrow \Sigma$, where $\Sigma$ is a closed, connected, oriented surface, such that $\pi$ is injective on the set of critical points $C=\left\{x_{1}, \ldots, x_{n}\right\}$ and about each $x_{i}$ and $\pi\left(x_{i}\right)$ there are complex local coordinate charts agreeing with the orientations of $M$ and $\Sigma$ on which $\pi$ is of the form

$$
\pi\left(z_{1}, z_{2}\right)=z_{1}^{2}+z_{2}^{2} \text {. }
$$

Any fiber containing a critical point is called a singular fiber. Clearly all regular fibers are closed surfaces and are of the same diffeomorphism type. We will assume that the generic fiber is connected and our fibration is relatively minimal, i.e., no fiber contains a $(-1)$ sphere. If the genus of the fiber is at least two, then a Lefschetz fibration is determined by its monodromy representation

$$
\pi_{1}\left(\Sigma-\left\{\pi\left(x_{1}\right), \ldots, \pi\left(x_{n}\right)\right\}\right) \rightarrow \mathcal{M}_{g} .
$$

(See 4 for more about Lefschetz fibrations.)

Recall that for a group $G$, the first homology group with integral coefficient is $H_{1}(G)=G /[G, G]$, where $[G, G]$ is the commutator subgroup of $G$, the subgroup generated by the commutators $[x, y]=x y x^{-1} y^{-1}$ for all $x, y \in G$.

Let $\Sigma_{g}$ be a closed oriented surface. It is well known that $H_{1}\left(\mathcal{M}_{g}\right)$ is generated by the class of a Dehn twist about a nonseparating simple closed curve and is equal to $\mathbb{Z}_{12}$ if $g=1, \mathbb{Z}_{10}$ if $g=2$ and trivial if $g \geq 3$. We say that a simple closed curve on a closed surface is nontrivial if it does not bound a disc.

Lemma 2.2. For every $h \geq 0, N(1, h)=12$.

Proof. All nontrivial simple closed curves on $\Sigma_{1}$ are nonseparating and the right Dehn twists map to the same generator under the natural map $\mathcal{M}_{1} \rightarrow H_{1}\left(\mathcal{M}_{1}\right)$. Hence, if a product of right Dehn twists is trivial (or equal to a product of commutators), then the number of twists is divisible by 12 . This implies that the number of singular fibers in a genus one Lefschetz fibration must be divisible by 12 . Moreover, 12 is clearly realized by the elliptic surfaces $E(1) \# \Sigma_{1} \times \Sigma_{h}$ for any $h \geq 0$. (Here \# denotes the fiber sum.)

The next result is due to Matsumoto [10].

Proposition 2.3. For every $h \geq 0,5 \leq N(2, h) \leq 8$.

Proof. Since every right Dehn twist about a nontrivial separating simple closed curve on $\Sigma_{2}$ is the product of 12 right Dehn twists about nonseparating simple closed curves, its image under the map $\mathcal{M}_{2} \rightarrow H_{1}\left(\mathcal{M}_{2}\right)$ is 2 . Hence, if a product of right Dehn twists about $n$ nonseparating and $s$ separating simple closed curves is trivial (or equal to a product of commutators), then

$$
n+2 s \equiv 0(\bmod 10)
$$

Therefore, there are at least 5 singular fibers in a genus two Lefschetz fibration over a closed surface. Moreover, a genus 2 Lefschetz fibration with 8 singular fibers is constructed in [10]. 
For a 4-manifold $Y$, let $e(Y)$ and $\sigma(Y)$ denote the Euler characteristic and the signature of $Y$, respectively. The next proposition is proved by Stipsicz [12], [14].

Proposition 2.4. Let $M$ be a 4-manifold which admits a relatively minimal genus $g>0$ Lefschetz fibration over a torus. Then $0 \leq c_{1}^{2}(M) \leq 10 \chi(M)$, where $c_{1}^{2}(M)=$ $3 \sigma(M)+2 e(M)$ and $\chi(M)=(\sigma(M)+e(M)) / 4$.

Proposition 2.5. $N(g, 1)>1$ for all $g \geq 1$.

Proof. Suppose that $N(g, 1)=1$ for some $g$. Then there is a 4-manifold $Y$ which admits a relatively minimal genus $g$ Lefschetz fibration over a torus with only one singular fiber. Hence $e(Y)=1$. The inequality $0 \leq c_{1}^{2}(Y)=3 \sigma(Y)+2 e(Y)$ implies that $\sigma(Y) \geq-2 / 3$. This gives $\sigma(Y) \geq 0$ since $\sigma(\bar{Y})$ is an integer.

On the other hand, $\chi(Y)=(\sigma(Y)+e(Y)) / 4=(\sigma(Y)+1) / 4$. Therefore, $\sigma(Y)=$ $4 \chi(Y)-1$. So we have $c_{1}^{2}(Y)=3 \sigma(Y)+2 e(Y)=12 \chi(Y)-1 \leq 10 \chi(Y)$. Hence $\chi(Y) \leq 0$ and $\sigma(Y) \leq-1$, which is a contradiction. This proves the proposition.

Corollary 2.6. The Dehn twist about a nontrivial simple closed curve on a closed surface cannot be equal to a single commutator.

Proof. Suppose that a right Dehn twist is a commutator. Then there is a relatively minimal Lefschetz fibration of genus $g$ with only one singular fiber over the torus. Hence, $N(g, 1)=1$, which contradicts Proposition 2.5.

We are now ready to prove our main results.

Proof of Theorem 1.2. Consider a sphere $X$ with four holes with boundary components $a, a_{1}, a_{2}, a_{3}$. By the lantern relation [5] there are three simple closed curves $b_{1}, b_{2}, b_{3}$ on $X$ such that

$$
t_{a} t_{a_{1}} t_{a_{2}} t_{a_{3}}=t_{b_{1}} t_{b_{2}} t_{b_{3}}
$$

or

$$
t_{a}=t_{b_{1}} t_{a_{1}}^{-1} t_{b_{2}} t_{a_{2}}^{-1} t_{b_{3}} t_{a_{3}}^{-1}
$$

Here for two diffeomorphisms $\alpha$ and $\beta$, the composition $\alpha \beta$ means that $\alpha$ is applied first. However, we will use the notation $\alpha(x)$ to mean that $\alpha$ is applied to $x$.

Since the genus of $\Sigma_{g}$ is at least three, $X$ can be embedded in $\Sigma_{g}$ in such a way that $a_{1}, a_{2}, a_{3}, b_{1}, b_{2}, b_{3}$ are all nonseparating. The simple closed curve $a$ can be chosen either nonseparating or separating bounding a subsurface of arbitrary genus (cf. Figure 1 and Figure 21). Furthermore, the complement of $a_{1} \cup b_{1}$ and that of $a_{2} \cup b_{2}$ are connected. Hence, there is an orientation preserving diffeomorphism $F$ of $\Sigma_{g}$ such that $F\left(a_{1}\right)=b_{2}$ and $F\left(b_{1}\right)=a_{2}$. Let $K$ be another orientation preserving diffeomorphism of $\Sigma_{g}$ such that $K\left(b_{3}\right)=a_{3}$ and let $f$ and $k$ be the isotopy classes of $F$ and $K$ respectively. Then

$$
\begin{aligned}
t_{a} & =t_{b_{1}} t_{a_{1}}^{-1} t_{F\left(a_{1}\right)} t_{F\left(b_{1}\right)}^{-1} t_{b_{3}} t_{K\left(b_{3}\right)}^{-1} \\
& =t_{b_{1}} t_{a_{1}}^{-1} f^{-1} t_{a_{1}} t_{b_{1}}^{-1} f t_{b_{3}} k^{-1} t_{b_{3}}^{-1} k \\
& =\left[t_{b_{1}} t_{a_{1}}^{-1}, f^{-1}\right]\left[t_{b_{3}}, k^{-1}\right] .
\end{aligned}
$$

This concludes the proof of Theorem 1.2. 

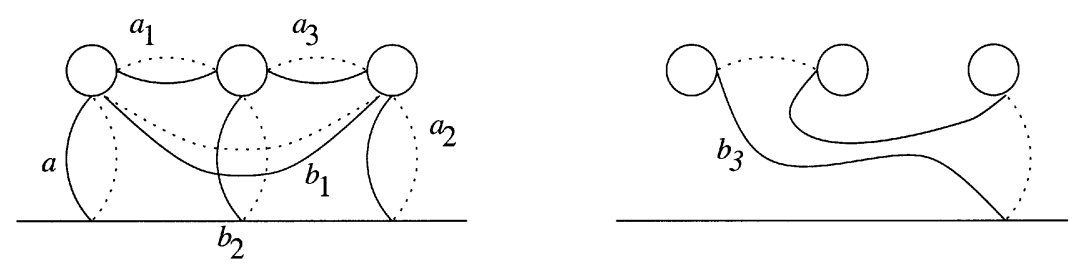

FiguRE 1. $a$ is nonseparating.
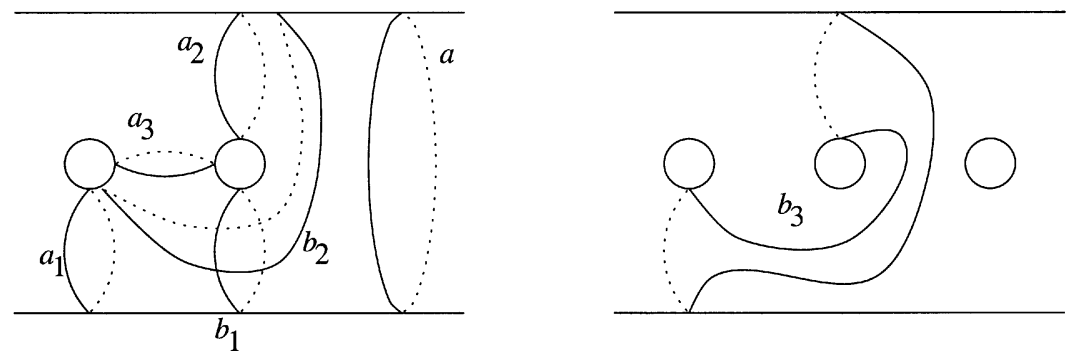

FiguRE 2. $a$ is separating.

Proof of Theorem 1.1. The fact that $N(g, h)>1$ is proved above for $g<3$ or $h=1$. Clearly, $N(g, 0)>1$ for all $g \geq 1$. Suppose that $g \geq 3$ and $h \geq 2$. Let $p$ and $q$ be two distinct points in $\Sigma_{h}$. Choose a free basis $\left\{x_{1}, y_{1}, x_{2}, y_{2}, \ldots, x_{h}, y_{h}\right\}$ of $\pi_{1}\left(\Sigma_{h} \backslash\{p\}, q\right)$ such that $\prod_{i=1}^{h}\left[x_{i}, y_{i}\right]$ is isotopic to a simple loop encircling the point $p$. Consider the following monodromy representation

$$
\Psi: \pi_{1}\left(\Sigma_{h} \backslash\{p\}, q\right) \rightarrow \mathcal{M}_{g},
$$

where

$$
\begin{gathered}
\Psi\left(x_{1}\right)=t_{b_{1}} t_{a_{1}}^{-1}, \\
\Psi\left(y_{1}\right)=f^{-1}, \\
\Psi\left(x_{2}\right)=t_{b_{3}}, \\
\Psi\left(y_{2}\right)=k^{-1},
\end{gathered}
$$

and if $h \geq 3$,

$$
\Psi\left(x_{i}\right)=\Psi\left(y_{i}\right)=1
$$

for $3 \leq i \leq h$.

This representation gives a genus $g$ Lefschetz fibration with only one singular fiber (whose vanishing cycle is the given curve $a$ ) over a closed surface of genus $h$ since we have the relation

$$
t_{a}=\left[t_{b_{1}} t_{a_{1}}^{-1}, f^{-1}\right]\left[t_{b_{3}}, k^{-1}\right] .
$$

Hence, $N(g, h)=1$ if $g \geq 3$ and $h \geq 2$.

This finishes the proof of the theorem. 
Remarks. 1) Theorem 1.2 answers a question of Mess in the negative. (See Problem 2.13 in [6].) If the genus of the surface is at least three, then the proof of Theorem 1.2 shows that $t_{a}^{n}$ can be expressed as a product of $(3 n+1) / 2($ resp. $3 n / 2)$ commutators if $n$ is odd (resp. even). Using the lantern relation, we can also prove that if the genus is at least three, then $t_{a}^{n}$ can be written as a product of $n$ (resp. $\left.n+1\right)$ commutators if $n$ is even (resp. odd).

2) The conclusions of Theorem 1.2 and Corollary 2.6 hold also for surfaces with boundary.

3) Theorem 1.2 also shows that the unique singular fiber can be chosen reducible or irreducible. (A singular fiber is called reducible (resp. irreducible) if the corresponding vanishing cycle is separating (resp. nonseparating)).

4) ESTIMATES ON $N(g, 0)$.

In [2], Cadavid proves that $N(g, 0) \leq 2 g+4$ if $g$ is even and $N(g, 0) \leq 2 g+10$ if $g$ is odd. (This result was also discovered independently by the first author [7].) Recently, Stipsicz 13 proved that $\frac{1}{5}(4 g+2) \leq N(g, 0)$.

5) ESTIMATES ON $N(2, h)$.

The second author showed that $N(2,0)=7$ or 8 in his thesis [11. It is also clear that there is a $k \geq 1$ such that $N(2, h)=5$ for each $h \geq k$ (cf. [3]).

6) Examples of a Lefschetz fibration with a unique singular fiber and prescribed fundamental group were constructed in [1].

\section{REFERENCES}

1. J. Amoros, F. Bogomolov, L. Katzarkov, T. Pantev, (with an appendix by Ivan Smith), Symplectic Lefschetz fibrations with arbitrary fundamental groups, e-print: GT/9810042.

2. C. Cadavid, Ph.D. Dissertation, UT Austin, 1998.

3. H. Endo, Meyer's signature cocyle and hyperelliptic fibrations, preprint.

4. R. Gompf and A. Stipsicz, 4-manifolds and Kirby calculus, Graduate Studies in Math., Amer. Math. Soc., Providence, RI, 1999. CMP 2000:01

5. D.L. Johnson, Homeomorphisms of a surface which act trivially on homology, Proc. Amer. Math. Soc. 75 (1979), 119-125. MR 80h:57008

6. R. Kirby, Problems in Low-Dimensional Topology, AMS/IP Stud. Adv. Math., 1997, 35-473. CMP 98:01

7. M. Korkmaz, Noncomplex smooth 4-manifolds with Lefschetz fibrations, preprint.

8. T-J. Li, Symplectic Parshin-Arakelov inequality, preprint.

9. Y. Matsumoto, Diffeomorphism types of elliptic surfaces, Topology 25 (1986), 549-563. MR 88b:32061

10. Lefschetz fibrations of genus two - a topological approach, Proceedings of the 37th Taniguchi Symposium on Topology and Teichmüller Spaces, ed. Sadayoshi Kojima et al., World Scientific (1996), 123-148. CMP 99:06

11. B. Ozbagci, Ph.D. Dissertation, UC Irvine, 1999.

12. A. Stipsicz, Chern numbers of certain Lefschetz fibrations, Proc. Amer. Math. Soc., to appear. CMP 99:01

13. - On the number of vanishing cycles in Lefschetz fibrations, Math. Res. Lett. 6 (1999), 449-456. CMP 2000:01

14. —_ Erratum to "Chern numbers of certain Lefschetz fibrations", preprint.

Department of Mathematics, Middle East Technical University, 06531 Ankara, TURKEY

E-mail address: korkmaz@math.metu.edu.tr

Department of Mathematics, University of California, Irvine, California 92697

E-mail address: bozbagci@math.uci.edu

Current address: Department of Mathematics, Michigan State University, Lansing, Michigan 48824

E-mail address: bozbagci@math.msu.edu 